(GMT) to adenovirus for persons with cases during sessions one and two (GMT 14 and GMT 28, respectively) were each significantly higher $(p<.01)$ than the GMT of persons not meeting the case definition (GMT 6). Bacterial analysis of grab samples of water obtained from the pond yielded 80 colonies per $100 \mathrm{cc}$ of fecal coliiorms, 200 colonies per $100 \mathrm{cc}$ of Enterococcus, and 9,000 colonies per $100 \mathrm{CC}$ of Staphylococcus. A concentrated sample of pond water drawn approximately six feet below the surface yielded adenovirus serotype 3. Residual chlorine was not detectable.

One week after the end of the second session the pond was drained, and most counselors left. No further outbreaks were reported following the second session; however, all subsequent sessions during the summer and fall were of maximum one-week duration.

\section{AVAILABILITY OF VARICELLA VACCINE FOR CHILDREN WITH ACUTE LYMPHOCYTIC LEUKEMIA}

An investigational, live, attenuated varicella vaccine is available free through Merck Research Laboratories to any physician requesting it for certain pediatric patients (aged 12 months to 17 years) with acute lymphocytic leukemia (ALL). Patients must meet specified criteria, including no clinical history of varicella and continuous remission for at least 12 months.
Varicella vaccine is being provided to this group of patients for use through a study protocol to monitor and evaluate safety. An investigational new drug application for the vaccine has been filed with the Food and Drug Administration.

Previous experience with this vaccine has shown it to be immunogenic in children with ALL. ${ }^{1}$ The most common reaction to the vaccine is mild (fewer than 100 lesions) varicelli-form rash, occurring in approximately $40 \%$ of vaccinees. ${ }^{2}$

The physician must provide information outlined in the protocol, and the protocol and consent form for the study must be approved by the institutions Investigation Review Board. Additional information about eligibility criteria and vaccine administration is available from Dr. Jo White, Merck Research Laboratories, telephone (215) 834-2554.

\section{REFERENCES}

1. Gershon AA, Steinberg SP, Varicella Vaccine Collaborative Study Group of the National Institute of Allergy and Infectious Diseases. Persistence of immunity to varicella in children with leukemia immunized with live attenuated varicella vaccine. $\mathrm{N}$ Engl JMed.1989;320:892-897.

2. Lydick E, Kuter BJ, Zajac BA, Guess HA, Varicella Vaccine Collaborative Study Group of the National Institute of Allergy and Infectious Diseases. Association of steroid therapy with vaccine-associated rashes in children with acute lymphocytic leukaemia who received Oka/Merck varicella vaccine. Vaccine. 1989;7:549-553. 